\title{
The 36 Strategies of the Chinese: Can It Be a Eurasian Strategic Model in a Global Context?
}

\author{
Prof. Dr. İsmail Melih Baş (Beykent University, Turkey)
}

\begin{abstract}
Although the 36 Strategies are a summary of some of the war strategies used by the ancient Chinese warriors, over the years, these have been commonly cited and used by many Chinese businessmen. These strategies are grouped under six categories. Each category contains six strategies. The six categories in turn can be used in two types of situations. While the three categories are used in a winning situation; the other three are used in a disadvantageous situation. However, the application and usage of these strategies can be mingled in various combinations. The set of these strategies is a very good alternative to the western style of management for global strategic competition for the Eurasian companies. This seems right both theoretically and practically. After the collapse of globalism of the 20th century, we are on the threshold of a new model of globalization. In that sense, while developing a Eurasian strategic management style, these 36 strategies of the Chinese provide a very good source, parallel to the market domination of Chinese economy in the global economy.
\end{abstract}

\section{Introduction}

The changing world order or the changing global economy of the twenty-first century may be titled as Eurasian Era. Perhaps the discussion in last Davos Summit the criticisms about globalisation by the western side especially USA, while Xi Jinping (president of PRC) defends a new order of globalisation. BRICS or the acronyms alike related to the Asia are the buzzwords of the economic press and literature as anyone in the street knows. According to the GDP (Gross Domestic Product) based on PPP (Purchasing Power Parity) rating of the PRC (People's Republic of China) was the first, and the USA was the second in 2016, surprisingly! Once upon a time China was the interesting market and the production center with an advantage of cheap labour for the western countries. But recently PRC companies' acquisitions even in USA is rapidly increasing. Trumponomics is characterized with a concept of new nationalism as new protectionism. These discussions are very common in the field of macroeconomics and macropolitics.

But, international business management with an Asian perspective at micro level has not been broadly discussed neither in the domains of academia nor in professional practice. Besides verbal culture, professional books and textbooks titled with these words are yet remain to be just a few, e.g. the three books written by those different authors Charles W. Hill (Hill,2012) etc.

According to George Yip and Bruce McKern, the history-making development of the Chinese economy has entered a new phase. China is moving aggressively from a strategy of imitation to one of innovation. Driven both by domestic needs and by global ambition, China is establishing itself at the forefront of technological innovation. Western businesses need to prepare for a tidal wave of innovation from China that is about to hit Western markets, and Chinese businesses need to understand the critical importance of innovation in their future (Yip and McKern,2016).

At this point, there is a question that needs to be considered: What is the strategic management culture of those Asian companies which they can use in the business? There are a few studies on this subject like Musashi's Five Rings (Japan), the 36 Strategies (China) etc.

In this proceeding, the 36 Strategies will be introduced, if this set is sufficient to be used as a Eurasian style of strategic management.

A short history first; the 36 Strategies are the war strategies used by the ancient Chinese warriors. The name of the collection comes from the Book of Qi, in its seventh biographical volume, Biography of Wang Jingze. Wang was a general who had served Southern Qi since the first Emperor Gao of the dynasty. Wang used thirty-six with a reference to the I Ching where six is the number of Yin that shared many characteristics with the dark schemes involved in military strategy. As 36 is the square of six, it therefore acted as a metaphor for numerous strategies. It is known as the original hand-copied paperback discovered and put into print by a local Publisher in 1941. The 36 Strategems only came to public's attention after its review was published in the Chinese Communist Party's Guangming Daily newspaper on September 16, 1961. It was subsequently reprinted and distributed with growing popularity.

After years, these strategies have been handled as business strategies in the light of metaphoric business wars. Although some books have been printed since 1992, a number of books published annually has not exploded (see the list at the end of this study). In that sense Prof. Harro von Senger (Senger, 1995) is a top profile. He translated the 36 Strategies into German. He also wrote a book the 36 Strategies for Business (Senger,2005). Professor Dr. Wee Chow Hou (Singapore) is also one of the names which must be remembered in 'the 36 strategies and business' topic (Hou, 1998). 
Perhaps Alibaba (by Jack Magic) as a remarkable Chinese company which is changing the face of global business must be analyzed in the light of the 36 strategies as well as say the Tencent company (Ersiman,2015; Tse 2015; Yiğ McKern 2016).

\section{The 36 Strategies: A Short View with Explanations and Examples as Titles}

These strategies are grouped under six categories. Each category contains six strategies. The six categories in turn can be used in two types of situations. While the three categories are used in a winning situation; the other three categories are used in a disadvantageous situation. However, the application and usage of these strategies can be mingled in various combinations (Senger 1995, Senger 2005, Verstappen, 1999, Hou and Luh, 1998).

\section{The Strategies Used in Winning Situation}

\section{Advantageous Strategies}

These strategies are used in situations when related resources and time are to one's advantage. Detailed planning can be carried out, because of there is no need to rush. The six strategies in this category will be handled below one by one with some illustrations:

\subsection{Deceiving the Heavens to Cross the Sea (Man tain guo tai).}

Explanation: It means to create a false impression to distract the target and so achieve one's goal without his knowledge. This strategy Works on the assumption that people take extra precautions when faced with new or difficult situations, but tend to let their guards down in familiar situations. Every one expects a secret to be hidden, so if one conducts an important plan openly, it may not be noticed. As the saying goes, 'An open situation hides a dark secret'. This strategy requires that the target is not alerted to what might happen. So even if the scheme was uncovered, it would be too late for him to take counteraction (Senger 1995, Senger, 2005, Yip and Mc Kern, 2016; De Monte; 2013; Lan,2014, Cleary, 1992, Baş, 2007).:

Examples (Taylor, 2013; Tse, 2015; Yip and Mc Kern, 2016; De Monte; 2013; Lan, 2014).:

- Managing change:

People typically resist change, more so if they have been operating within their comfort zones or in a stable environment. To lure them out of their comfort zones, their defense mechanisms must be lowered through the use of non-threatening situations, and by introducing gradual changes. A good example of this is the effort put in by Singapore Telecoms in its privatization efforts. It began preparing its whole organization at least six years before the company was listed. Changes were introduced gradually so as to minimize any negative impact on the morale of the personnel. Their efforts paid off.

- $\quad$ Adoption of products and services:

This strategy applies to marketing too. A market may be resistant to new and radical products, especially if these are high-ticket items or items that are difficult to operate. To overcome resistance, marketers may use one or a combination of the following methods:

Free trial period with Money-back guarantee / Free or low-cost lessons on usage of the products /services (For example golf clubs in Asia typically conduct low-cost lessons to lure potential participants) / Allowing the product to be bought piecemeal / Selling a basic unit with options for upgrading or add-ons / Special offers - packages (The sale of foreign properties by western companies to the more affluent Asian consumers provides a good example).

- $\quad$ Product / Service Bundling and Tying Contracts

Pricing the whole bundle lower than sum of the individual prices of each product /service, the marketer makes a product or service more attractive. As products get more sophisticated, they are sold more and more as bundles. After paying so much for the complete product bundle, many consumers do not fully use all the benefits or features offered.

Bundling is widely used by Japanese manufacturers of cars, consumer electronics, watches and appliances.

- $\quad$ Financing for expansion

Occasionally, a company may need funds for expansion. Funds can be obtained by borrowing from the bank or from shareholders. In order to entice shareholders, the company may price the rights below the market price of share so as to make them very attractive. The company may be able to meet its financial needs while making shareholders happy.

\section{- $\quad$ Management of services}

This strategy resolves round the use of distractions to lure the target from one's main concerns. These nonthreatening distractions can take many forms, and are created to appeal to the desire for creature comforts.

The way in which Singapore airlines (SIA) strives to create an enjoyable experience for its passengers is fascinating. SIA's success has led other airlines, especially those in Asia to adopt similar strategies. 


\subsection{Besieging Wei to Save Zhao.}

Explanation: Instead of attacking a cohort of strong enemies, one should try to diffuse the concentration and attack each enemy separately. Instead of a head-on attack, one should wait for the best opportunity when the enemy is at his weakest point to launch an ambush (Senger 1995, Senger, 2005, Yip and Mc Kern, 2016; De Monte; 2013; Lan,2014, Cleary, 1992, Baş, 2007).

Examples (Taylor, 2013; Tse, 2015; Yip and Mc Kern, 2016; De Monte; 2013; Lan,2014).:

- Changing the point(s) of contact

- Choosing areas ignored by competitors

- Creating one's own advantages

\subsection{Killing with a Borrowed Knife.}

Explanation: This strategy works on the premise that while the enemy has clarified its stand, the position of the alliance is still equivocal. In this situation, the best way is to entice the alliance to destroy the enemy on one's behalf. This is an adaptation of the logic in harm theory: harm the bottom to benefit the top (Senger 1995, Senger, 2005, Yip and Mc Kern, 2016; De Monte; 2013; Lan,2014, Cleary, 1992, Baş, 2007).

Examples (Taylor, 2013; Tse, 2015; Yip and Mc Kern, 2016; De Monte; 2013; Lan,2014).:

- $\quad$ Operating through surrogates

- $\quad$ Relying on foreign investments to stimulate growth

- $\quad$ Licensing, franchising and joint-ventures

\subsection{Conserving Energy While the Enemy Tires Himself Out.}

Explanation: One does not need to always make a direct attack to subdue the enemy. Sometimes, one can use the theory of 'using the mild (or weak) to control the strong to delay and exhaust the enemy and strengthen one's position (Senger 1995, Senger, 2005, Yip and Mc Kern, 2016; De Monte; 2013; Lan,2014, Cleary, 1992, Baş, 2007).

Examples (Taylor, 2013; Tse, 2015; Yip and Mc Kern, 2016; De Monte; 2013; Lan,2014).:

- $\quad$ Strike when the timing is right

- $\quad$ Capitalizing on falling prices

- $\quad$ Entering foreign markets

\subsection{Looting A House on Fire.}

Explanation: When the enemy is in a big crisis, that is the time to destroy him. This will enable the strong to conquer the weak (Senger 1995, Senger, 2005, Yip and Mc Kern, 2016; De Monte; 2013; Lan,2014, Cleary, 1992, Baş, 2007).

Examples (Taylor, 2013; Tse, 2015; Yip and Mc Kern, 2016; De Monte; 2013; Lan,2014).:

- Vulnerability created by internal squabbles

- $\quad$ Exploiting chaotic business situations

- Currency and stock market raiders

\subsection{Making A Feint to The East but Hitting Out in The West.}

Explanation: When the enemy is in a state of confusion, like grass growing wild, he will not be able to clearly assess a situation. Neither will he able to cope with sudden and unexpected changes in circumstances. A good strategist defeats the enemy by taking advantage of him when he has lost control (Senger 1995, Senger, 2005, Yip and Mc Kern, 2016; De Monte; 2013; Lan,2014, Cleary, 1992, Baş, 2007).

Examples (Taylor, 2013; Tse, 2015; Yip and Mc Kern, 2016; De Monte; 2013; Lan,2014).:

- Concealing one's true intentions

\section{Advantageous Strategies Offensive Strategies}

Like advantageous strategies, these strategies also are used in situations when time and resources are not constraining factors. However, these strategies seek to gain victory through direct attack.

\subsection{Hitting the Grass to Startle the Snake}

Explanation: An attempt should be made to clarify suspicious circumstances before making the next move. To repeatedly probe and investigate a suspicious matter is the best way to discover the enemy's plot (Senger 1995, Senger, 2005, Yip and Mc Kern, 2016; De Monte; 2013; Lan,2014, Cleary, 1992, Baş, 2007).

Examples (Taylor, 2013; Tse, 2015; Yip and Mc Kern, 2016; De Monte; 2013; Lan,2014).:

- The art of smiling in winning business 


\subsection{Hitting the Grass to Startle the Snake Borrowing a Corpse to Resurrect a Soul}

Explanation: A person that is of use may be more difficult to take advantage of our assistance. A less useful (or weaker) person may request our assistance. When I am able to use the weak one to my advantage, I am giving him my strength at his request (Senger 1995, Senger, 2005, Yip and Mc Kern, 2016; De Monte; 2013; Lan,2014, Cleary, 1992, Baş, 2007).

Examples (Taylor, 2013; Tse, 2015; Yip and Mc Kern, 2016; De Monte; 2013; Lan,2014).

- Operating under the umbrella of others

- Going overseas

- $\quad$ Extending product life cycle

- $\quad$ Sell-outs, mergers and acquisitions

\subsection{Luring A Tiger from Its Lair in The Mountain}

Explanation: Trap the enemy when the natural elements are to his disadvantage. Then create false impressions to lure him out. A direct attack may prove dangerous. It is far better to lure the enemy out of his comfort and destroy him (Senger 1995, Senger, 2005, Yip and Mc Kern, 2016; De Monte; 2013; Lan,2014, Cleary, 1992, Baş, 2007).

Examples (Taylor, 2013; Tse, 2015; Yip and Mc Kern, 2016; De Monte; 2013; Lan,2014).

- Choosing the site of negotiation

- $\quad$ Luring important foreign investments

- $\quad$ Attracting foreign talents

\subsection{Releasing the Enemy to Recapture Him Later}

Explanation: Sometimes, it is not advisable to drive the enemy into a corner as he may retaliate. The objective is not to destroy him completely as there may be good reason to keep him alive. It is better to let the enemy escape and tire himself out while pursuing closely behind. The enemy will be soon be exhausted and will lose his fighting spirit. The best time to capture him is when he has fallen apart, without bloodshed. In business war, sheer physical dominance does not ensure victory. More important is the need to win the enemy's hearts. In this way, one can secure total surrender and loyalty (Senger 1995, Senger, 2005, Yip and Mc Kern, 2016; De Monte; 2013; Lan,2014, Cleary, 1992, Baş, 2007).

Examples (Taylor, 2013; Tse, 2015; Yip and Mc Kern, 2016; De Monte; 2013; Lan,2014).:

- $\quad$ Exhaust the resources of the competitors

- Use of varied strategies

- Winning the heart takes time

\subsection{Tossing Out a Brick to Get a Jade}

Explanation: Use a decoy to entice the enemy, get him muddled and he will fall into the trap (Senger 1995, Senger, 2005, Yip and Mc Kern, 2016; De Monte; 2013; Lan,2014, Cleary, 1992, Baş, 2007).

Examples (Taylor, 2013; Tse, 2015; Yip and Mc Kern, 2016; De Monte; 2013; Lan,2014).

- Use of baits in business

- Investment incentives

- Auctions for charity

\subsection{Disband the Bandits by Arresting Their Leader}

Explanation: If the enemy's power-base is destroyed in an attack and its leader is captured, the whole organization will break down. Just like a dragon from the sea wanting to stage a war on land, it will face severe difficulties (Senger 1995, Senger, 2005, Yip and Mc Kern, 2016; De Monte; 2013; Lan,2014, Cleary, 1992, Baş, 2007).

Examples (Taylor, 2013; Tse, 2015; Yip and Mc Kern, 2016; De Monte; 2013; Lan,2014).: Corporate head hunting / Head hunting for academics and researchers / Getting to the root of the problem

\section{Deception Strategies}

Deception is commonly used in war to create advantageous for oneself, and to handicap the enemy. Such strategies are designed to mislead the enemy.

\subsection{Replace Superior Beams and Pillars with Inferior Ones}

Explanation: Find a chance to change the enemy's battle array frequently, and take away its main source of power. Wait till it is unable to cope with the situation before attacking it. Pillars and beams are important structures in a building. The quality pf the pillars and beams determine the stability of the building. The arrangement of a 
company's physical and human assets has parallel significance (Senger 1995, Senger, 2005, Yip and Mc Kern, 2016; De Monte; 2013; Lan,2014, Cleary, 1992, Baş, 2007).

Examples (Taylor, 2013; Tse, 2015; Yip and Mc Kern, 2016; De Monte; 2013; Lan,2014).: Short changing the consumer / Burden of proof on the consumers / Pirated goods / Making consumers aware of replacements

\subsection{Pointing at The Mulbery But Scolding the Locust Tree.}

Explanation: A stronger force can use warning or admonishment to control a lesser force. A proper display of power will receive support and the use of a decisive method will demand reverence. This strategy is similar to the Chinese saying, 'Kill the chicken to scare the monkeys' (Senger 1995, Senger, 2005, Yip and Mc Kern, 2016; De Monte; 2013; Lan,2014, Cleary, 1992, Baş, 2007).

Examples (Taylor, 2013; Tse, 2015; Yip and Mc Kern, 2016; De Monte; 2013; Lan,2014).:

Severe punishments on offenders / As a warning to others / Being in a position of strength

\subsection{Pretending to Be Insane but Remaining Smart.}

Explanation: It is better for one to pretend that he knows nothing and take no action rather than to pretend that he knows everything and rush into a situation hastily. One should prepare his forces in the dark and not let others know of his secret and wait for an opportunity to strike, just as lightning and thunder wait to strike in winter. To use this strategy, one has to perfect the art of deception because it takes discipline to foreign weakness when one is really strong. It requires self-imposed humility, something which few can muster. Yet when one is able to achieve such a skill, he is able even to 'pretend to be a pig to prey on the tiger' (Senger 1995, Senger, 2005, Yip and Mc Kern, 2016; De Monte; 2013; Lan,2014, Cleary, 1992, Baş, 2007).

Examples (Taylor, 2013; Tse, 2015; Yip and Mc Kern, 2016; De Monte; 2013; Lan,2014).:

Business tycoons and top executives / Self-enforced humility / Fakery and illusions

\subsection{Remove the Ladder After the Enemy Ascends to The Roof.}

Explanation: Pretend to expose one's weakness to lure the enemy into one's trap. When the enemy is within one's control, cut off its supply routes and eventually destroy him. This strategy warns against taking a small advantage when one can go for the killing as that may result in one's destruction. The key to this strategy is the 'ladder'. This ladder acts as the bait to arouse the curiosity of the enemy. Once the enemy is up on the roof, the ladder is removed, trapping the enemy (Senger 1995, Senger, 2005, Yip and Mc Kern, 2016; De Monte; 2013; Lan,2014, Cleary, 1992, Baş, 2007).

Examples (Taylor, 2013; Tse, 2015; Yip and Mc Kern, 2016; De Monte; 2013; Lan,2014).:

Creating advantages for oneself / Incremental trapping

\subsection{Deck the Tree with Flowers}

Explanation: Borrow a setting to boost one's image. Although one may be on the weak side, one's battle array can appear strong (Senger 1995, Senger, 2005, Yip and Mc Kern, 2016; De Monte; 2013; Lan,2014, Cleary, 1992, Baş, 2007).

Examples (Taylor, 2013; Tse, 2015; Yip and Mc Kern, 2016; De Monte; 2013; Lan,2014).: The art of packaging and branding / Packaging of services / Private residential properties

\subsection{The Guest Takes Over as Host.}

Explanation: Try to put a foot in when there is a crack and eventually, control the brain of the enemy (Senger 1995, Senger, 2005, Yip and Mc Kern, 2016; De Monte; 2013; Lan,2014, Cleary, 1992, Baş, 2007).

Examples (Taylor, 2013; Tse, 2015; Yip and Mc Kern, 2016; De Monte; 2013; Lan,2014).:

Foot-in-the-door approach / Involving labour in management / Profit-sharing and stock ownership schemes

\section{The Strategies Used in Disadvantageous Situations}

\section{Opportunistic Strategies}

These strategies thrive on situations where vulnerabilities can be exploited. The idea is to capitalize on all opportunities so s to gain the advantage.

\subsection{Creating Something Out of Nothing}

Explanation: The strategy advocates using a false front, not to deceive the enemy totally, but to make what is false seem real. If one can use false fronts to conceal the truth, the enemy will have a misconception about a situation. That is the time to launch an attack (Senger 1995, Senger, 2005, Yip and Mc Kern, 2016; De Monte; 2013; Lan,2014, Cleary, 1992, Baş, 2007).

Examples (Taylor, 2013; Tse, 2015; Yip and Mc Kern, 2016; De Monte; 2013; Lan,2014).: 
Bidding of favorite / popular numbers / Advertising and sales promotion / Targeting and positioning / Share buyback schemes

\subsection{Secret Escape Through Chen Cang.}

Explanation: The gist of this strategy is to hit the enemy at the point where he is least prepared, that is, his back. In battle, one should pretend to expose one's movement, then launch a surprise attack at the enemy's weakest point, when he is ill-prepared to defend himself (Senger 1995, Senger, 2005, Yip and Mc Kern, 2016; De Monte; 2013; Lan,2014, Cleary, 1992, Baş, 2007).

Examples (Taylor, 2013; Tse, 2015; Yip and Mc Kern, 2016; De Monte; 2013; Lan,2014).:

Contingency planning in business / Use of shell and project-based companies

\subsection{Observing the Fire from The Other Side of The River.}

Explanation: When there are disorder and internal struggles amongst the enemy's forces, one should wait till the enemy's situation turns bad before attacking. Arguments and unhappiness in the enemy's camp, weaken the enemy's strength. This is when one should seize the opportunity and reap the benefits with minimal effort (Senger 1995, Senger, 2005, Yip and Mc Kern, 2016; De Monte; 2013; Lan,2014, Cleary, 1992, Baş, 2007).:

Examples (Taylor, 2013; Tse, 2015; Yip and Mc Kern, 2016; De Monte; 2013; Lan,2014).:

Squabbles within family business / Exploiting conflicts in international business / Staying out of uncertain situations

\subsection{A Dagger Sheathed in a Smile.}

Explanation: One should win the trust of one's enemy so as to disarm him while preparing to launch an ambush. The enemy must not be alerted to the changes that have taken place and the ambush must only be made when one is well-prepared. This fulfils the saying, 'What appears to be weak (or soft) on the outside may actually be strong (or hard) on the inside (Senger 1995, Senger, 2005, Yip and Mc Kern, 2016; De Monte; 2013; Lan,2014, Cleary, 1992, Baş, 2007).

Examples (Taylor, 2013; Tse, 2015; Yip and Mc Kern, 2016; De Monte; 2013; Lan,2014).:

The art of smiling in winning business

\subsection{The Plum Dies in Place of the Peach.}

Explanation: Where losses are inevitable, one should be willing to make sacrifices to gain victory. In warfare, this strategy is applied when the enemy has the upper hand. To turn the tables around and achieve victory, one needs to make sacrifices. It may be necessary to sacrifice a few battles to achieve the ultimate objective of winning the war (Senger 1995, Senger, 2005, Yip and Mc Kern, 2016; De Monte; 2013; Lan,2014, Cleary, 1992, Baş, 2007).

Examples (Taylor, 2013; Tse, 2015; Yip and Mc Kern, 2016; De Monte; 2013; Lan,2014).

Prioritizing the use of limited resources / Focusing on the right markets / Sacrifices needed

\subsection{Stealing A Goat Along the Way.}

Explanation: No matter how small a hole may be; one should use it to one's advantage. No matter how small the advantage is, one should obtain it for his benefit. One should take advantage of the enemy's small weakness for one's small gain. This is similar to the analogy that by gathering crumbs of bread, one can still get a full meal. However, if one is only interested in the full loaf, there may be none at all (Senger 1995, Senger, 2005, Yip and Mc Kern, 2016; De Monte; 2013; Lan,2014, Cleary, 1992, Baş, 2007).

Examples (Taylor, 2013; Tse, 2015; Yip and Mc Kern, 2016; De Monte; 2013; Lan,2014).:

Seizing every business opportunity / Growing a business / Taking on more than one can chew / Opportunities are not limited by situations

\section{Confusion Strategies}

These strategies aim to confuse a pursuing enemy to throw him off his guard. In this way, valuable time can be gained in making one's escape.

\subsection{Pulling Out the Firewood from Beneath the Cauldron.}

Explanation: When faced with a strong opponent, direct confrontation may not be advisable. It may be better to use tactics to destroy his morale (Senger 1995, Senger, 2005, Yip and Mc Kern, 2016; De Monte; 2013; Lan,2014, Cleary, 1992, Baş, 2007).

Examples (Taylor, 2013; Tse, 2015; Yip and Mc Kern, 2016; De Monte; 2013; Lan,2014).:

Removing key corporate lieutenants / Major accounts and anchor tenants / Avoid head-on competition 


\subsection{Catching A Fish in Troubled Waters}

Explanation: The literal meaning of the strategy is to take advantage of the troubled or muddy situation in which the fish is unable to see clearly and has restricted movement. In such circumstances, catching the fish by hand may be good enough. This strategy is similar to the Chinese saying, 'Throwing a rock down a well after the enemy has fallen in'. When the enemy is in the well, his movements are restricted, thus it is easy to destroy him (Senger 1995, Senger, 2005, Yip and Mc Kern, 2016; De Monte; 2013; Lan,2014, Cleary, 1992, Baş, 2007).

Examples (Taylor, 2013; Tse, 2015; Yip and Mc Kern, 2016; De Monte; 2013; Lan,2014).:

Avoiding confusion / Exploiting chaotic situations

\subsection{Catching A Fish in Troubled Waters Making an Unnoticed Escape Like a Golden Cicada Shedding Its} Skin.

Explanation: By preserving the original formation of an army and the strength of its force, one will not arouse the suspicion of the allies. And the enemy will not be roused to action. However, in actual fact, one secretly diverting his main strength to attack the enemy from another direction (Senger 1995, Senger, 2005, Yip and Mc Kern, 2016; De Monte; 2013; Lan,2014, Cleary, 1992, Baş, 2007).

Examples (Taylor, 2013; Tse, 2015; Yip and Mc Kern, 2016; De Monte; 2013; Lan,2014).:

Contingency planning / Withdrawal of expatriate professionals / Divesting / Reducing investment \& equity / Taking a company to public listing

\subsection{Shutting the Doors to Catch the Thief.}

Explanation: The best way to destroy a weak enemy is to first have him surrounded, like shutting off all routes of escape, when catching a thief (Senger 1995, Senger, 2005, Yip and Mc Kern, 2016; De Monte; 2013; Lan,2014, Cleary, 1992, Baş, 2007).

Examples (Taylor, 2013; Tse, 2015; Yip and Mc Kern, 2016; De Monte; 2013; Lan,2014).:

Handling weaker products and brands / Exploiting the advantage of size / Zero-sum outcome

\subsection{Befriend the Far and Attack the Near.}

Explanation: Sometimes, because of geographical constraints, it is more beneficial to attack a nearby enemy than to attack another far away. If the situation permits, form a temporary alliance with a distant enemy to destroy the enemy nearby (Senger 1995, Senger, 2005, Yip and Mc Kern, 2016; De Monte; 2013; Lan,2014, Cleary, 1992 , Baş, 2007).

Examples (Taylor, 2013; Tse, 2015; Yip and Mc Kern, 2016; De Monte; 2013; Lan,2014).:

Strategic alliance with distant partners / International trade and investment / Franchising and licensing

\subsection{Borrow A Passage to Attack Guo.}

Explanation: A smaller state situated between the enemy and oneself should be given immediate support if the enemy threatens to control it. In this way, one will earn the trust of the smaller state and may eventually exert one's influence over the latter. Mere words without action will not win the trust of a small force in a precarious situation (Senger 1995, Senger, 2005, Yip and Mc Kern, 2016; De Monte; 2013; Lan,2014, Cleary, 1992, Baş, 2007).

Examples (Taylor, 2013; Tse, 2015; Yip and Mc Kern, 2016; De Monte; 2013; Lan,2014).:

Marketing under other companies' brands / Backward / Forward integration and piggybacking / Diversifying through acquisitions / Listing companies on foreign stock exchange

\section{Desperate Strategies}

In a desperate situation, one may have to resort to unconventional and unorthodox methods and means. As such, some of these strategies can be quite dramatic and tragic. However, when used appropriately, such strategies can be very effective.

\subsection{Beauty Scheme.}

Explanation: With regards to an enemy with strong troops, one should aim to control its general. Against an intelligent enemy general, one should plot to dampen his morale. Once the general's fighting spirit is quenched, the army will be weakened. Therefore, one should try to hit the enemy at its weaknesses while conserving one's energy (Senger 1995, Senger, 2005, Yip and Mc Kern, 2016; De Monte; 2013; Lan,2014, Cleary, 1992, Baş, 2007).

Examples (Taylor, 2013; Tse, 2015; Yip and Mc Kern, 2016; De Monte; 2013; Lan,2014).:

Good looks are the assets / Reality in business / Importance of training and grooming

\subsection{Empty City Scheme.}

Explanation: There is no fixed method for deploying an army. It may sometimes be better to deliberately display its weakness, to confuse the enemy and make the latter abandon the attack for fear of trickery. In a situation where 
the enemy is strong and when one is weak, proper use of this strategy can appear extraordinary (Senger 1995, Senger, 2005, Yip and Mc Kern, 2016; De Monte; 2013; Lan,2014, Cleary, 1992, Baş, 2007).

Examples (Taylor, 2013; Tse, 2015; Yip and Mc Kern, 2016; De Monte; 2013; Lan,2014).:

A high-risk strategy / Putting up a brave front / Use of bogus companies and swindling syndicates

\subsection{Double Agent Ploy.}

Explanation: When the enemy lays traps, set a trap within his traps to create internal chaos. When one secures assistance from within the enemy's ranks, one will not lose the battle. The literal meaning of the strategy is to use the enemy's spy to counter spy on the enemy (Senger 1995, Senger, 2005, Yip and Mc Kern, 2016; De Monte; 2013; Lan,2014, Cleary, 1992, Baş, 2007).

Examples (Taylor, 2013; Tse, 2015; Yip and Mc Kern, 2016; De Monte; 2013; Lan,2014).:

Industrial and commercial espionage / Consultants, research companies \& agents / Protecting the business

\subsection{Self-Injury Scheme.}

Explanation: No one would intentionally harm himself. Therefore, if someone is hurt, others would believe that he has been injured by another. Pretense will thus gain the enemy's trust and sow discord among enemy members. This is similar to playing with an innocent child (Senger 1995, Senger, 2005, Yip and Mc Kern, 2016; De Monte; 2013; Lan,2014, Cleary, 1992, Baş, 2007).

Examples (Taylor, 2013; Tse, 2015; Yip and Mc Kern, 2016; De Monte; 2013; Lan,2014).:

Price discounts and sales promotion / Overcoming short-term difficulties / Cutting losses and gaining market share

\subsection{A Series of Interconnected Ploys.}

Explanation: If the enemy has a strong and powerful army, a head-on confrontation is not advisable. Instead, one should utilize a few connected strategies to decrease the enemy's power. With a good leadership and careful planning, it will be easy to win the battle as if heaven is on one's side (Senger 1995, Senger, 2005, Yip and Mc Kern, 2016; De Monte; 2013; Lan,2014, Cleary, 1992, Baş, 2007).

Examples (Taylor, 2013; Tse, 2015; Yip and Mc Kern, 2016; De Monte; 2013; Lan,2014).:

Corporate and marketing strategies (using a combination of different strategies) / Multiple strategies in business (The case of Kia Motors) / Innovate and create advantages

\subsection{Escape - The Best Scheme.}

Explanation: In a situation where the enemy is obviously stronger, total retreat may be the best option. This strategy advocates retreating as a means to plan for the next attack (Senger 1995, Senger, 2005, Yip and Mc Kern, 2016; De Monte; 2013; Lan,2014, Cleary, 1992, Baş, 2007).

Examples (Taylor, 2013; Tse, 2015; Yip and Mc Kern, 2016; De Monte; 2013; Lan,2014).: Doing business in a corrupt environment / Operating in unfamiliar markets / Knowing when to quit

\section{The Question: Can It Be a Eurasian Strategic Model in a Global Context?}

This question seems very rational, if we consider that we are in the Eurasian Age. If it is so, a second level analysis appears: what are the factors which effect it.

\subsection{Supporting Factors}

a. Eurasian memetics (cultural genetics); b. Rising new-nationalism; c. New protectionism; d. Falling capitalism and the values related; e. Uprising of BRICS but specially China in economic competition field; $f$. Confucius Institutes affiliated with Ministry of Education of PRC; f. Others (Senger 1995, Senger, 2005, Yip and Mc Kern, 2016; De Monte; 2013; Lan,2014, Cleary, 1992, Baş, 2007).

\subsection{Inhibiting Factors}

a. Wrong habits in training in the universities (adopting only westernized strategic thinking, strategic management - westernization as globalisatorship). The 36 strategies are not included in top list Strategic Management textbooks in Turkish and in English as well. There is a Porterization (Michael Porter as a guru from Harvard University) trend; $b$. Cultural resistance (memetics?) to socialist countries including PRC (People's Republic of China) as a result of cold war mentality and 'the end of history - Fukuyama' false effect in politics; c. The golden era of capitalism (1945 + approx. three decades) and the socio-economic hallo effect of this on business strategic management style; d. Weakness of cultural relation efforts by PRC in that sense with the developing countries; e. The censorship in the management literature in the capitalist countries especially The USA (Harvard University etc.), United Kingdom (London School of Economics-LSE, London Business School-LBS etc.); d. Others (Senger 1995, Senger, 2005, Yip and Mc Kern, 2016; De Monte; 2013; Lan,2014, Cleary, 1992, Baş, 2007). 


\section{Recommendations (To Do List)}

\subsection{Academic Field}

Symposiums: International Business Strategic Management with an Asian perspective (why not?); publications, works like textbooks (not anything in Turkish yet), articles; researches works: thesis, professional researches supported by the institutions, e.g. Studies on Alibaba, Tencent etc. in the light of the 36 Strategies; consultancy works: university-industry joint projects (Senger 1995, Senger, 2005, Yip and Mc Kern, 2016; De Monte; 2013; Lan,2014, Cleary, 1992, Baş, 2007).

\subsection{Practice (Industry and Services)}

Adopting another course of action by our national companies SME's (Small and Medium Enterprises first): the 36 strategies by PRC or Five Rings by Japan instead of Portermania (as Obsessive-Compulsive Disorder); training and consultancy supports by the state especially SMBA (Small and Medium Business Administration - KOSGEB in Turkey) (Senger 1995, Senger, 2005, Yip and Mc Kern, 2016; De Monte; 2013; Lan,2014, Cleary, 1992, Baş, 2007).

\section{References}

- Baş, 2007. "Stratejik Yönetimde Asyagil Bir Araç: Çinlilerin 36 Strategemi”, Bilim ve Gelecek Dergisi, 44, p.22-30.

- Lan, 2014. The 36 Ancient Chinese Strategies for Modern Business, LAC International Press, Atlanta.

- Cleary, 1992. The Book of Leadership and Strategy: Lessons of the Chinese Masters, Shambhala.

- De Mente, 2013. The Chinese Way in Business: Secrets of Succesful Business Dealings in China, Tuttle Publishing, North Clarendon, VT.

- Ersiman, 2015. Alibaba's World, Macmillan, London.

- Hill, et. al, 2012. International Business an Asian Perspective, McGraw Hill, New York.

- Hou and Luh, 1998. The 36 Strategies of the Chinese, Singapore, Addison Wesley, Reading Massachusetts.

- Senger, 1993. The Book of Strategems, Penguin Books, (Translated into Turkish and published - titled as Savaş Hileleri - as 3 volumes by Anahtar Kitaplar Pub.Co. first in 2006, and in 2014 again).

- Senger, 2005. The 36 Stratagems for Business: Achieve Your Objectives Through Hidden and Unconventional Strategies and Tactics, Cyan Communications, (Translated into Turkish and published titled as Yöneticiler İçin 36 Strategem - by Anahtar Kitaplar Pub. Co. in 2007).

- Taylor, 2013. The Thirty-Six Stratagems: A Modern Interpretation of a Strategy Classic (Infinite Success), Infinite Ideas, Oxford.

- Tse, 2015. China Disruptors: How Alibaba, Xiaomi, Tencent, and Other Companies Changing the Rules of Business, Portfolio, London.

- Verstappen, 1999. The Thirty-six Strategies of Ancient China, China Books \& Periodicals, South San Francisco, CA.

- Yip and McKern, 2016. China's Next Strategic Advantage: From Imitation to Innovation, The MIT Press, Cambridge Massaschusetts. 